\title{
ADAPTACIÓN Y ESTUDIO PSICOMÉTRICO DE DOS INSTRUMENTOS DE PAREJA: ÍNDICE DE SATISFACCIÓN MATRIMONIAL Y ESCALA DE INESTABILIDAD MATRIMONIAL
}

\author{
loseba Iraurgi, Mireya Sanz ${ }^{1}$ y Ana Martínez-Pampliega \\ Deusto-Salud. Unidad I + D + I en Psicología Clínica y de la Salud. Universidad de Deusto, España \\ (RECibido el $12 / 11 / 2009$, Aceptado el 20/12/2009)
}

\begin{abstract}
RESUMEN
El presente estudio analiza las características psicométricas de los instrumentos de pareja: Índice de Satisfacción Matrimonial - ISM (Booth y Edwards, 1983) y Escala de Inestabilidad Matrimonial - EIM (Hudson, 1992). Ambos instrumentos son aplicables a situaciones de crisis familiares y han resultado ser eficaces a la hora de predecir el divorcio o la separación. En este estudio se utilizó una muestra formada por 183 mujeres. Se destacan las sólidas propiedades psicométricas de ambas escalas, con coeficientes de fiabilidad altos (alphas de 0,90 y 0,97 para el ISM y EIM, respectivamente), correcta validez estructural (validada mediante análisis factorial confirmatorio), y adecuada validez concurrente (coeficientes de correlación por encima de 0,40 en constructos afines). Todo ello permite considerar los instrumentos adaptados al castellano como útiles para su aplicación. Se discute, no obstante, la necesidad de comprobar su bondad métrica en diferentes muestras, contextos y problemáticas clínicas de pareja.
\end{abstract}

Palabras clave: Familia, pareja, Índice de Satisfacción Matrimonial, Escala de Inestabilidad Matrimonial, características psicométricas.

\section{ABSTRACT \\ ADAPTATION AND PSYCHOMETRIC STUDY OF TWO INSTRUMENTS OF COUPLE: INDEX OF MARITAL SATISFACTION AND MARITAL INSTABILITY SCALE}

This study examines the psychometric characteristics of the instruments of partner: Index of Marital Satisfaction - IMS (Booth and Edwards, 1983) and Marital Instability Scale - EIM (Hudson, 1992). Both instruments are applicable to situations of family crisis and have proved effective in predicting divorce or separation. We used a sample consisting of 183 women. It highlights the strong psychometric properties of both scales, with high reliability coefficients (alphas 0.90 and 0.97 for the ISM and EIA, respectively), correct structural validity (validated by confirmatory factor analysis), and adequate concurrent validity (correlation coefficients above 0.40 in related constructs). All this allows adaptation of both spanish valuation couple

1 Director de la Unidad de Investigación, Desarrollo e Innovación en Psicología Clínica y de la Salud Adscrita a la Universidad de Desusto (España). E-mail: ioseba_iraurgi@deusto.es 
as useful for their application. It argues, however, the need to test their goodness metrics in different samples, contexts and clinical problems of couples.

Keywords: Family, couple, Index of Marital Satisfaction, Marital Instability Scale, psychometric characteristics.

\section{INTRODUCCIÓN}

Uno de los campos donde se pone de manifiesto la carencia de instrumentos fiables, válidos y sobre todo bien adaptados a nuestra cultura, es el del conflicto matrimonial. Si bien es frecuente la utilización de instrumentos que recogen el ajuste o la satisfacción matrimonial, como el Test de Ajuste Matrimonial -Marital Adjustment Test- (Locke-Wallace, 1959), el PREPARE (Olson, Fournier y Druckman, 1986), la Escala de Satisfacción Marital (Pick y Andrade, 1988), la Escala de Satisfacción Marital (Roach, Frazier y Bowden, 1981) o la Escala de Ajuste Diádico (DAS, Spanier, 1976), es mucho más difícil encontrar instrumentos aplicables al estudio de la separación o divorcio, sobre todo si deseamos que sean breves, psicométricamente robustos, relevantes a nivel internacional y que estén bien adaptados culturalmente a nuestro país (Reig-Ferrer, Cepeda-Benito y Snyder, 2004; Martínez-Pampliega, 2008). A este respecto, con la pretensión de dar un pequeño paso en esa dirección, el presente artículo plantea la adaptación de dos instrumentos: la Escala de Inestabilidad Matrimonial (Booth and Edwards, 1983) y el Índice de Satisfacción Matrimonial (Hudson, 1992), aplicables a situaciones de crisis familiares y que han resultado ser eficaces a la hora de predecir el divorcio o la separación.

La Escala de Inestabilidad Matrimonial -Marital Instability Scale- (Booth y Edwards, 1983) es un instrumento de cinco ítems dirigido a predecir la ruptura de un matrimonio. Fue desarrollado para ayudar a evaluar la inestabilidad entre las parejas intactas, con independencia de su calidad matrimonial. Es decir, permite diferenciar qué parejas, con baja calidad matrimonial, se separarán y quiénes no.

La segunda escala es el Índice de Satisfacción Matrimonial (Index of Marital Satisfaction), fue desarrollada por Hudson (1992) con el fin de medir el grado, la severidad o la magnitud de un problema que un cónyuge o su pareja tiene en la relación matrimonial. El cuestionario describe diez situaciones que recogen aspectos representativos de la vida de una pareja en temas generales como relaciones sexuales, manifestaciones de afecto o interés, comunicación, tiempo libre o tareas caseras. La conflictividad que se presenta en las distintas situaciones se centra en: ser ignorado en demandas o planteamientos respecto a temas concretos, enfrentarse a desacuerdos, demandar realización de tareas cuyo cumplimiento es desagradable o poco grato, ser criticado, ser insultado, menospreciado o ignorado.

Los dos instrumentos han demostrado tener una adecuada sensibilidad tanto en investigación como en la práctica clínica, a juzgar por los estudios en los que se encuentran presentes dentro del panorama internacional. La Escala de Inestabilidad Emocional ha sido empleada en estudios dirigidos a analizar el impacto de factores adversos, o por el contrario, tras el desarrollo de programas de intervención. Por ejemplo, el grupo de Matthews (Matthews, Wickrama y Conger, 1996) vinculó la hostilidad y el afecto en las historias de parejas y 
su divorcio o separación. Rueter, Conger y Ramisetty (1999) analizaron la inestabilidad de diversos programas de intervención sobre las pautas de socialización. También ha sido empleada para comprobar el impacto de factores como la tensión económica (Johnson y Booth, 1990), en la estabilidad de pareja. Por otro lado, el índice de satisfacción matrimonial se encuentra presente en estudios dirigidos a medir la relación entre disolución de la relación y calidad matrimonial (Leone y Hall, 2003), las relaciones familiares (Schuchts y Witkin, 1989) o las problemáticas familiares como el abuso infantil (Mollerstrom, Patchner y Milner, 1992). En todos estos estudios ambas escalas han ofrecido adecuadas características psicométricas, mostrándose, asimismo, como herramientas idóneas que deberían formar parte de los recursos disponibles al servicio de la práctica clínica o de la investigación. No obstante, una revisión de las bases de datos nos muestra la práctica ausencia de cualquier intento de adaptación de estos instrumentos al castellano. Tan sólo señalamos el estudio desarrollado en México por Escamilla (2006) en el que el Índice de Satisfacción Matrimonial mostró altos niveles de fiabilidad (coeficiente alpha de Cronbach de 0,90$)$.

El objetivo del estudio será lograr la adaptación cultural de los instrumentos, para lo cual se tendrán en cuenta cinco tipos de equivalencia entre la escala original y la escala adaptada: Equivalencia conceptual (investigar qué dominios son importantes para el concepto en la cultura objetivo y las relaciones entre ellos), Equivalencia de ítems (examinar los ítems empleados para esos dominios, los cuales podrían variar entre culturas), Equivalencia semántica (asegurarse de que la traducción lleva a la equivalencia semántica de los ítems), Equivalencia operacional (asegurarse de que los métodos de medida empleados son apropiados para la cultura en cuestión) y Equivalencia de medida (examinar los resultados del proceso en términos del comportamiento del instrumento), para la cual contamos con las orientaciones de Hambleton, Merenda y Spielberger (2005).

\section{MÉTODO}

\section{Participantes}

La muestra está formada por 183 mujeres, todas ellas con hijos de edades comprendidas entre 8 y 18 años de edad. En su mayoría fueron familias con uno $(29,5 \%, \mathrm{~N}=54)$ o dos hijos $(56,8 \%, N=104)$. Sólo en un 28,9\% $(\mathrm{N}=53)$ las participantes habían cursado carreras medias o superiores; en un $30,1 \%(\mathrm{~N}=55)$ los estudios correspondían a ESO, Bachiller o FP y en un 27,9\% ( $\mathrm{N}=51)$ a estudios primarios. Con respecto a su profesión en su mayoría eran trabajadoras por cuenta ajena $(45,5 \%, \mathrm{~N}=84)$, seguido de amas de casa $(31,7 \%, \mathrm{~N}=58)$.

\section{Instrumentos de medida}

- Escala de Inestabilidad Matrimonial (Marital Instability Scale - Booth y Edwards, 1983).

Como se ha indicado previamente, se trata de un instrumento de 5 ítems dirigido a predecir la ruptura del matrimonio. La escala en su versión completa consta de 19 
ítems sobre aspectos cognitivos y conductuales: pensar en el divorcio, hablar sobre encontrar un trabajo, volver a estudiar, hablar con personas significativas sobre el divorcio, reuniones con consejeros sobre la posibilidad de divorcio y separación física del esposo, etc. La escala completa presenta una fiabilidad de 0,93; la escala reducida con tan sólo 5 ítems, aunque presenta una fiabilidad algo más baja, sigue siendo aceptable: 0,75 . La validez fue demostrada a través de dos procedimientos: jueces externos y correlaciones con variables relacionadas (raza, religión, residencia, empleo y disolución matrimonial).

- Índice de Satisfacción Matrimonial (Index of Marital Satisfaction - Hudson, 1982).

Esta escala consta de 25 ítems y fue desarrollada por Hudson (1992) con el fin medir el grado, la severidad o la magnitud de un problema que un esposo o su pareja tiene en la relación matrimonial. Presenta diversos puntos de corte con el fin de identificar ausencia o presencia de problemas significativos a nivel clínico, e incluso la presencia de violencia en la relación. Es un instrumento de autoinforme, fácil de administrar y se puede aplicar en varias ocasiones para comprobar cambios en la intensidad del problema de la pareja. Posee cinco opciones de respuesta (de rara vez o nunca a muchas veces o siempre). Este instrumento puede ser respondido independientemente del tipo de relación de pareja, del estatus clínico, del nivel de formación o de la cultura de los cónyuges. Su nivel de fiabilidad es muy alto, habiéndose encontrado un alpha de Cronbach de 0,96. También presenta una buena validez tanto convergente como discriminante y de constructo. Por otro lado, ha sido vinculado con criterios externos significativos (satisfacción matrimonial o problemas matrimoniales).

- Cuestionario de aserción en la pareja (ASPA - Carrasco, 1996).

Este instrumento fue desarrollado con el fin de evaluar cuatro tipos de estrategias de comunicación (aserción, agresión, sumisión y agresión pasiva) que cada miembro de la pareja puede poner en marcha a la hora de afrontar situaciones conflictivas que pueden surgir en una convivencia marital. El cuestionario describe diez situaciones que recogen aspectos representativos de la vida de una pareja en temas generales como relaciones sexuales, manifestaciones de afecto o interés, comunicación, tiempo libre o tareas caseras. El estudio psicométrico del cuestionario fue realizado sobre 418 parejas, y se encontró una fiabilidad de 0,$83 ; 0,81 ; 0,75$ y 0,84 para las cuatro subescalas (aserción, agresividad, sumisión y agresión pasiva). Los análisis factoriales del instrumento y la correlación encontrada con el cuestionario DAS (Escala de Ajuste Diádico, Spanier, 1976) demostraron su validez de constructo. En este estudio, el análisis de fiabilidad de las cuatro dimensiones que componen el ASPA, así como la fiabilidad alcanzada por la escala total resultaron satisfactorios con coeficientes alpha de Cronbach entre 0,72 y 0,91 . El estilo de comunicación más representativo entre las mujeres evaluadas es de tipo asertivo $(M=3,81)$ y el menos expresado el agresivo $(\mathrm{M}=$ 1,98). Los índices de asimetría muestran valores aceptables salvo el caso del estilo agresivo con una clara asimetría positiva, es decir, la mayor frecuencia de puntuaciones se localizan en valores bajos (Tabla N. ${ }^{0} 1$ ). 
- $\quad$ Listado de Síntomas de Hopkins. Versión reducida (VR-LSH).

Es una escala empleada para analizar síntomas psicológicos, la cual fue desarrollada a partir del Listado de Síntomas de Hopkins (Hopkins Symptom Checklist, HSCL; Derogatis, Lipman, Rickels, Uhlenhuth y Covi, 1974). En este estudio hemos empleado una versión reducida de 30 ítems (Calvete y Villa, 2000). A través de los ítems se analizan cinco factores: somatización, depresión, ansiedad, dificultades cognitivas y sensibilidad interpersonal. En la adaptación inicial del instrumento (Calvete y Villa, 2000), la fiabilidad oscila entre 0,71 y 0,78 para las diferentes subescalas y 0,92 para la escala total. Más recientemente ha sido empleada por Muñoz (2003) en la realización de un estudio con población separada o divorciada. La fiabilidad encontrada fue incluso más alta a la indicada anteriormente. En el presente estudio tanto la escala total como cada una de las cinco dimensiones han mostrado índices de fiabilidad altamente satisfactorios con valores comprendidos entre 0,76 y 0,95 , de este modo, de muestra una alta consistencia interna del instrumento para medir su objetivo. Entre un mínimo y un máximo de 1 y 5 puntos, respectivamente, la media de sintomatología expresada por las madres evaluadas se sitúa en 2,27 puntos, con valores medios en las diferentes dimensiones entre 2,12 y 2,52; en ningún caso sobrepasan el valor 3 que indicaría la media del rango de sintomatología. La puntuación más alta se halla en la dimensión 'sensibilidad interpersonal' y las más bajas en 'somatización y ansiedad'. En definitiva, la expresión de síntomas de malestar emocional no es elevada, situándose en valores moderados-bajos (Tabla N. ${ }^{0} 1$ ).

- Escala de Satisfacción Familiar (Olson y Wilson, 1982).

Esta escala permite evaluar el grado de satisfacción con diez aspectos de la dinámica familiar, cinco de ellos vinculados con la cohesión y cinco con la adaptabilidad. El instrumento fue desarrollado en relación con el modelo circumplejo con el fin de cubrir una de sus hipótesis, la cual señala que es más importante la satisfacción que experimenta una familia sobre su nivel de cohesión y adaptabilidad, que el lugar donde se sitúa en el modelo. Originalmente la escala constaba de 14 ítems, pero posteriormente en 1989, Olson la redujo a 10 (Olson, Steward y Wilson, 1990), con los cuales se evalúa el grado de satisfacción experimentado con aspectos relacionados con la cohesión y adaptabilidad familiar. Podemos encontrar dos subescalas, satisfacción con la cohesión y satisfacción con la adaptabilidad, pero los autores consideran la escala global más válida y fiable. De hecho, el análisis factorial indicó claramente que se trataba de una escala unidimensional. Esta escala ha mostrado buenos criterios de fiabilidad tanto en la versión original (alpha de 0,91 y coeficiente test-retest de 0,75 ), como en la adaptación española (Sanz, 2003), presentando un alpha de Cronbach de 0,92. Respecto a la fiabilidad test-retest, se obtuvo un índice de 0,95. Similares resultados han sido obtenidos en estudios más recientes (Martínez-Pampliega, Sanz, Iraurgi et al., 2004). 
Tabla N. ${ }^{0}$ 1. Análisis de fiabilidad y características descriptivas de las dimensiones que componen el ASPA y el SCL-30.

\begin{tabular}{lcccccc}
\hline & No $^{\mathbf{i}}$ (ems & Rango & Media & $\begin{array}{c}\text { Desviación } \\
\text { típica }\end{array}$ & Asimetría & Fiabilidad \\
\cline { 2 - 7 } ASPA (Estrategias de comunicación) & & & & & & \\
Aserción & 10 & $1-5$ & 3,81 & 1,15 & $-0,16$ & 0,89 \\
Agresión & 10 & $1-5$ & 1,98 & 0,97 & 1,22 & 0,91 \\
Sumisión & 10 & $1-5$ & 2,44 & 0,71 & 0,68 & 0,72 \\
Agresión pasiva & 10 & $1-5$ & 2,39 & 0,85 & 0,43 & 0,84 \\
Total & 40 & $1-5$ & 2,66 & 0,47 & $-0,52$ & 0,79 \\
& & & & & & \\
SCL-30 (Listado de síntomas - Hopkins) & & & & & & \\
Somatización & 7 & $1-5$ & 2,12 & 0,77 & 0,67 & 0,84 \\
Depresión & 8 & $1-5$ & 2,40 & 0,79 & 0,30 & 0,88 \\
Ansiedad & 5 & $1-5$ & 2,13 & 0,73 & 0,53 & 0,80 \\
Dificultades cognitivas & 4 & $1-5$ & 2,46 & 0,76 & 0,10 & 0,76 \\
Sensibilidad interpersonal & 6 & $1-5$ & 2,52 & 0,80 & 0,07 & 0,88 \\
Total & 30 & $1-5$ & 2,27 & 0,68 & 0,26 & 0,95 \\
\hline
\end{tabular}

\section{Procedimiento}

Previamente al estudio de valoración psicométrica, se sometieron los instrumentos a un proceso de traducción, adaptación conceptual a nuestro contexto cultural y retrotraducción (Brislin, 1986; Behling y McFillen, 2000). En una primera fase, dos miembros del equipo tradujeron de forma independiente la versión original de la EIM y del ISM. Ambas traducciones fueron discutidas simultáneamente entre los traductores hasta alcanzar un consenso; asimismo, se evaluó la equivalencia conceptual y claridad del enunciado de los ítems hasta llegar a una versión adaptada. En una segunda fase, una persona nativa de EE.UU., con formación en psicología y que desconocía la versión original, tradujo la versión en castellano de nuevo al inglés. En una tercera fase, se comparó esta versión retrotraducida con la original a efectos de contrastar su equivalencia conceptual y de contenido, así como su consonancia sintáctica y técnica. Posteriormente, se aplicaron los instrumentos y se analizó el funcionamiento psicométrico de los cuestionarios, de esta forma se obtuvo la versión definitiva.

\section{Análisis de datos}

Para la descripción de las características de los ítems y de los indicadores de los instrumentos se utilizaron la frecuencia (n) y los porcentajes (\%) y/o los estadísticos de tendencia central (media, M y desviación estándar, DE), según el tipo de variables consideradas, nominales versus intervalo, respectivamente.

Para el estudio de la fiabilidad de los instrumentos se ha analizado la consistencia interna de cada uno de ellos y de sus dimensiones teóricas y empíricas a través del coeficiente alfa $(\alpha)$ de Cronbach (1951). Dicho coeficiente nos informa hasta qué punto todos los ítems de una escala miden una misma dimensión o, por el contrario, hasta qué punto miden 
cosas diferentes, pudiéndose corroborar de este modo su significación teórica (Nunnally y Berstein, 1995).

Con el objeto de estudiar la validez de constructo de los instrumentos se han utilizado dos estrategias de análisis. En primer lugar, se realizó un Análisis Factorial Exploratorio (AFE) de componentes principales con rotación varimax. Para comprobar el grado de interrelación de las variables, se calculó el índice Kaiser-Meyer-Olkin (KMO) y la prueba de esfericidad de Barlett $\left(\chi^{2}\right)$. Se consideraron aquellos factores/dimensiones con valores propios (Eigenvalue) superiores a 1. Para la interpretación de las dimensiones subyacentes, se consideraron aquellos ítems con una carga factorial con saturación superior a 0,40. La aplicación de esta estrategia de análisis AFE nos permite explorar las dimensiones subyacentes de los instrumentos y ver si éstas se aproximan a las teóricas.

Secundariamente, se realizó una secuencia de Análisis Factoriales Confirmatorios (AFC) a partir de técnicas estructurales de covarianza con objeto, en este caso, de contrastar o verificar las dimensiones de la formulación teórica sobre los instrumentos. Para ello, se estimó el grado de ajuste a los datos de la muestra a los modelos teóricos hipotetizados a través de la aplicación del programa EQS (Bentler, 1995; Bentler y Wu, 1995), utilizando para la estimación de los parámetros el método de máxima verosimilitud. Para evaluar el nivel de bondad de ajuste del modelo hipotetizado se dispone de diversos índices entre los que se encuentra el Ji cuadrado $\left(\chi^{2}\right)$. Este índice nos indica la probabilidad de que la divergencia entre la matriz de varianzas-covarianzas muestrales y la generada a partir del modelo hipotetizado sean debidas al azar, y por lo tanto un valor de probabilidad no significativo $(\mathrm{p}>0,05)$ sería indicativo de un buen ajuste (ambas matrices no difieren entre sí). Dado que el $\chi^{2}$ es muy sensible a las variaciones del tamaño de la muestra y a la violación de ciertos supuestos -linealidad, multinormalidad y aditividad- se han propuesto diversas medidas adicionales de la bondad de ajuste del modelo, entre las cuales se ha optado por las siguientes: 1) la razón entre el Ji cuadrado y los grados de libertad, 2) el índice de bondad de ajuste LISREL (GFI, Goodness of Fit Index), 3) el índice de ajuste normado de Bentler y Bonett (NFI, Normed Fit Index), y 4) la raíz cuadrada media de error de aproximación (RMSEA, Root Mean Square Error of Approximation). Para que exista un buen ajuste, la razón $\chi^{2} / \mathrm{gl}$ ha de ofrecer un valor menor de 3 , los valores GFI y CFI deben acercarse al valor unidad (preferiblemente >0,90), y los valores RMSEA deben ser menores o iguales a 0,08 -cuanto menor sea el valor, mejor ajuste- (Browne y Cudeck, 1993).

Por último, la validez concurrente ha sido estudiada a través del análisis de asociación del ISM y EIM con medidas de otros constructos con las que se espera que tenga relaciones significativas basándose en determinados modelos teóricos y la investigación previa realizada. Para su análisis se han calculado las correlaciones de Pearson (r) entre los instrumentos bajo estudio y los cuestionarios ASPA (Carrasco, 1996), VR-SLH 30 (Derogatis et al., 1974) y la satisfacción familiar (Olson y Wilson, 1982).

Todos los análisis, salvo el análisis factorial confirmatorio, se han realizado mediante el programa SPSS V.15 (Norusis, 2007). 
Tabla N. ${ }^{\circ}$ 2. Estadísticos descriptivos, análisis de fiabilidad y análisis factorial exploratorio y confirmatorio de la Escala de Inestabilidad Matrimonial.

\begin{tabular}{|c|c|c|c|c|c|c|c|}
\hline \multirow{3}{*}{ Ítems } & \multirow{2}{*}{\multicolumn{2}{|c|}{$\begin{array}{l}\text { Descriptivos } \\
\text { Rango (1-4) }\end{array}$}} & \multicolumn{2}{|c|}{ Fiabilidad } & \multicolumn{3}{|c|}{ Análisis factorial } \\
\hline & & & \multirow{2}{*}{$\begin{array}{l}\text { Correlación } \\
\text { ítem con el } \\
\text { resto de la } \\
\text { escala }\end{array}$} & \multirow{2}{*}{$\begin{array}{l}\text { Alpha } \\
\text { si se } \\
\text { elimina } \\
\text { el ítem }\end{array}$} & \multirow{2}{*}{$\begin{array}{l}\text { Exploratorio } \\
\text { Saturaciones }\end{array}$} & \multicolumn{2}{|c|}{ Confirmatorio } \\
\hline & Media & D.T. & & & & $\begin{array}{l}\text { Coeficiente } \\
\text { estructural }\end{array}$ & Error \\
\hline Considerar posibilidad de divorcio entre la pareja & 1,30 & 0,67 & 0,83 & 0,85 & 0,91 & 0,85 & 0,91 \\
\hline Hablar de separación / divorcio con amigos & 1,30 & 0,73 & 0,79 & 0,86 & 0,88 & 0,86 & 0,88 \\
\hline Considerar el matrimonio en crisis & 1,73 & 0,73 & 0,63 & 0,90 & 0,74 & 0,90 & 0,74 \\
\hline Considerar consulta con abogado & 1,17 & 0,54 & 0,68 & 0,89 & 0,79 & 0,89 & 0,79 \\
\hline \multirow[t]{3}{*}{ Plantearse separación o divorcio últimos tres años } & 1,30 & 0,64 & 0,84 & 0,85 & 0,91 & 0,85 & 0,91 \\
\hline & & & & & \multicolumn{3}{|c|}{ Índices de ajuste } \\
\hline & 1,36 & 0,56 & $\begin{array}{l}\text { Fiabilidad } \\
\text { escala total }\end{array}$ & 0,90 & $\begin{array}{c}\text { KMO }=0,87 \\
\chi^{2}=550 ; \\
\mathrm{p}<0,001 \\
\text { Eigenvalue: } 3,61 \\
\text { Var. Exp.: } 72,2 \% \\
\text { Coef. Theta }=0,90\end{array}$ & \multicolumn{2}{|c|}{$\begin{array}{c}\chi_{(5)}^{2}=4,01 ; \\
\mathrm{p}=0,547 \\
\chi^{2} / \mathrm{g} \cdot 1 .=0,82 \\
\text { RMSEA }=0,04 \\
\text { GFI }=0,99 \\
\text { CFI }=0,98\end{array}$} \\
\hline
\end{tabular}

\section{RESULTADOS}

\section{Escala de inestabilidad matrimonial}

Los datos descriptivos de los elementos de la escala, su fiabilidad y análisis de componentes principales se hallan en la Tabla N. ${ }^{\circ}$ 2. La escala de inestabilidad matrimonial está compuesta por cinco ítems cuyas puntuaciones se sitúan entre 1,17 y 1,73-con desviaciones estándar entre 0,54 y 0,73 - siendo la media de la escala total de 1,36 $(\mathrm{DE}=0,56)$ para un intervalo de puntuaciones entre 1 y 4 puntos. Todos los ítems presentan una alta contribución al conjunto de la escala (valores de correlación de entre 0,63 y 0,84 ) la cual alcanza un coeficiente de fiabilidad de 0,90 . La matriz de correlaciones de estos cinco ítems resulta susceptible de ser factorizada $(\mathrm{KMO}=0,87$; Prueba de esfericidad de Barlett estadísticamente significativa), obteniéndose una solución de un único factor que explica el 72,2\% de la varianza, obteniéndose, asimismo, un coeficiente theta de 0,90. Las cargas factoriales que componen el factor oscilan entre 0,74 y 0,91 , con contribución de todos los ítems componentes.

Dado que esta escala es de nueva adaptación al castellano, se realizó un segundo análisis factorial bajo una estrategia confirmatoria a fin de corroborar el constructo planteado desde la teoría que propone un único factor. En las dos columnas a la derecha de la Tabla N. ${ }^{\circ} 2$ se presentan los coeficientes estructurales y errores estándar de los elementos componentes y los índices de valoración del modelo. Todos los índices son reflejo de la bondad de ajuste de los datos empíricos al constructo teórico planteado; tanto el GFI como el NFI son 
muy elevados, por encima de 0,95 , el valor del Ji cuadrado entre sus grados de libertad se halla por debajo del valor 3 , y el error de aproximación cuadrático medio se sitúa por debajo del valor 0,08 . Por su parte, la magnitud de los coeficientes estructurales son en todos los casos elevados, por encima de 0,70 . Todos estos datos informan que los datos se ajustan al modelo teórico propuesto y, por tanto, la escala recoge apropiadamente el constructo que pretende medir.

Tabla N. ${ }^{0}$ 3. Matriz de correlaciones de las escalas de inestabilidad (EIM) y satisfacción (ISM) matrimonial con las escalas ASPA, SCL-30 y satisfacción familiar.

\begin{tabular}{lcccc}
\hline & \multicolumn{2}{c}{ Inestabilidad matrimonial } & \multicolumn{2}{c}{ Satisfacción matrimonial } \\
\cline { 2 - 5 } \multicolumn{1}{c}{ ASPA } & $\mathbf{r}$ & $\mathbf{p}$ & $\mathbf{r}$ & $\mathbf{p}$ \\
\cline { 2 - 5 } Asertividad & $-0,260$ & 0,008 & $-0,503$ & 0,001 \\
Agresividad & 0,604 & 0,001 & 0,567 & 0,001 \\
Sumisión & 0,056 & 0,495 & 0,278 & 0,001 \\
Agresividad pasiva & 0,451 & 0,001 & 0,602 & 0,001 \\
Total & 0,354 & 0,001 & 0,426 & 0,001 \\
& & & & \\
Somatización & 0,253 & 0,004 & 0,224 & 0,011 \\
Depresión & 0,275 & 0,002 & 0,367 & 0,001 \\
Ansiedad & 0,225 & 0,011 & 0,223 & 0,012 \\
Dificultades cognitivas & 0,158 & 0,082 & 0,208 & 0,021 \\
Sensibilidad interpersonal & 0,262 & 0,005 & 0,294 & 0,001 \\
Total & 0,285 & 0,005 & 0,314 & 0,001 \\
& & & & \\
Satisfacción familiar & $-0,435$ & 0,001 & $-0,438$ & 0,001 \\
r - Correlación de Pearson; $\mathbf{p}-$ Valor de probabilidad & & \\
\hline
\end{tabular}

En la Tabla N. ${ }^{\circ} 3$ se presenta la matriz de correlaciones entre la escala de inestabilidad matrimonial y otros constructos con los que se espera asociación. En lo que se refiere a la escala ASPA se observan correlaciones elevadas sobre todo con las subescalas de agresión activa $(0,60)$ y pasiva $(0,45)$. Es decir, a mayor puntuación en inestabilidad mayor tendencia al empleo de la agresión como medio de expresión, ya sea directa (empleo de coacciones, insultos, amenazas) o indirecta (manipulación, retirada del afecto, limitación de la comunicación). También la satisfacción familiar tiene una importante correlación con la estabilidad de pareja; a menor estabilidad peor satisfacción familiar. Respecto a la asociación con manifestaciones sintomáticas, se observan correlaciones estadísticamente significativas con casi todas las subescalas del SLH-30 (somatización, depresión, ansiedad y sensibilidad interpersonal). 
Tabla N. ${ }^{\circ}$ 4. Estadísticos descriptivos, análisis de fiabilidad y análisis factorial exploratorio de la escala de satisfacción matrimonial.

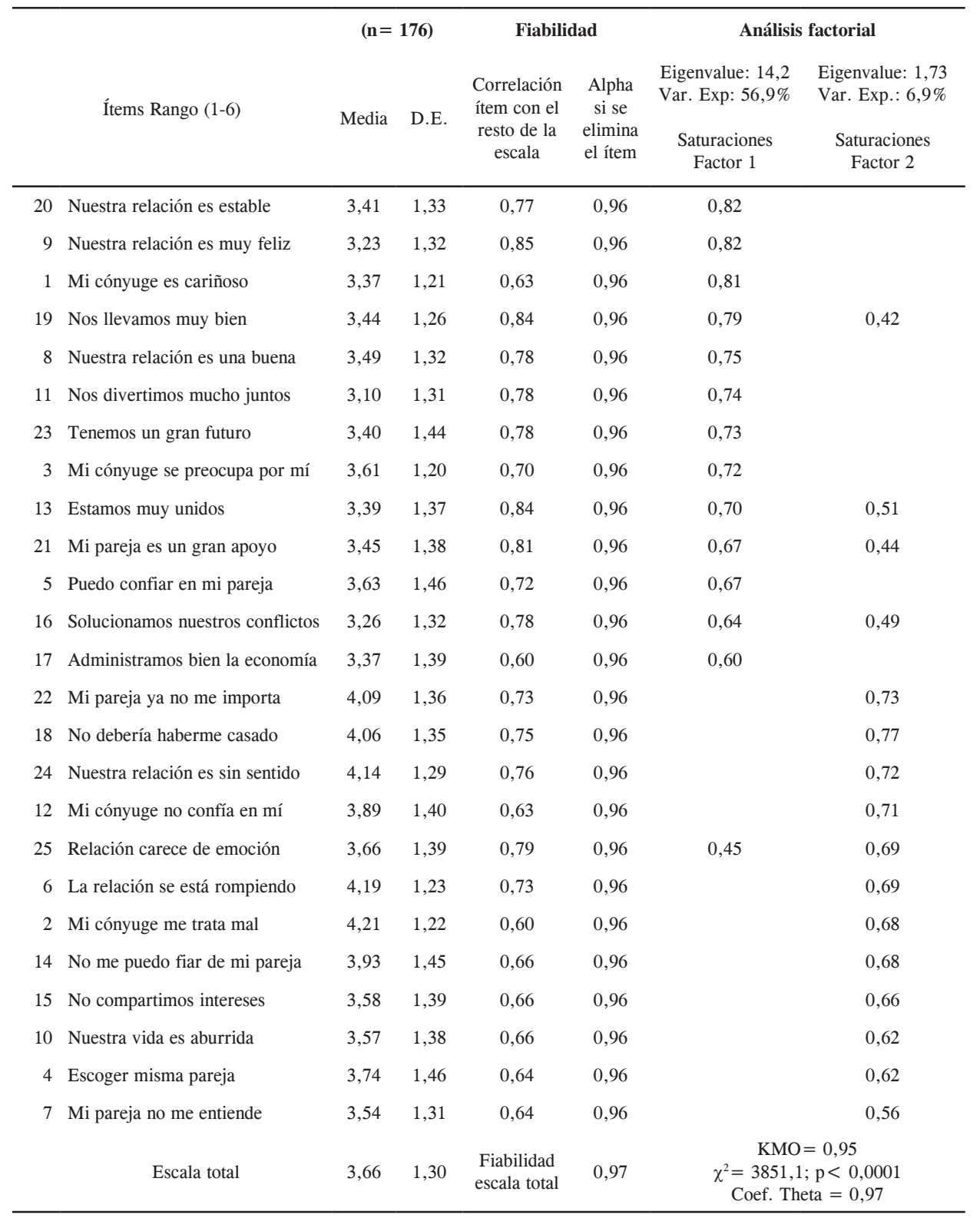




\section{Índice de satisfacción matrimonial (ISM)}

Los datos descriptivos de los elementos de la escala de satisfacción matrimonial, su fiabilidad y análisis de componentes principales se hallan en la Tabla N. ${ }^{\circ}$. Para un intervalo de puntuaciones entre 1 y 6 , los valores medios obtenidos se sitúan entre un mínimo de 3,10 y un máximo de 4,21 -con desviaciones estándar entre 1,20 y 1,46- siendo la media de la escala total de 3,66 (DE = 1,30). Los 25 ítems que componen el ISM presentan una alta contribución, en todos los casos, al conjunto de la escala (valores de correlación de entre $0,60$ y 0,85$)$ y que alcanza un coeficiente de fiabilidad casi perfecto (de 0,97$)$. La matriz de correlaciones de estos 25 ítems resulta susceptible de ser factorizada (KMO = 0,95; Prueba de esfericidad de Barlett estadísticamente significativa), obteniéndose una solución de dos factores que explican en conjunto el $63,8 \%$ de la varianza. El primer factor agrupa 13 ítems con cargas factoriales superiores a 0,60 y explica un 56,9\% de la varianza; el segundo factor, reúne 12 elementos con cargas superiores a 0,56 y explica en 6,9\% de la varianza. Cinco ítems son compartidos; cuatro elementos con cargas superiores en el factor 1 tendrían una contribución moderada en el factor 2, y un ítem componente del factor 2 tendría un peso moderado en el factor 1 . El examen de la composición de los factores nos permite observar que en el factor 2 se agrupan los elementos que han de ser recodificados dado que su enunciado está expresado en sentido inverso a la dimensión de satisfacción que se desea medir. Por tanto, podemos plantear que la diferenciación en dos factores se debe más a una diferenciación semántica que de la medida del constructo explorado.

Dado que este es otro instrumento adaptado, se ha procedido a su análisis mediante técnicas confirmatorias de análisis estructural de covarianzas. Como hemos podido apreciar, la alta contribución de todos los elementos a la consistencia interna de la escala y la alta fiabilidad alcanzada sugiere la posibilidad de plantear una única dimensión subyacente, como teóricamente venía propuesto. No obstante, el análisis exploratorio de componentes principales sugiere dos dimensiones, que por la distribución de sus elementos pudieran subsumirse en una dimensión global de satisfacción. Dados estos a priori se ha planteado la verificación mediante técnicas confirmatorias de dos modelos: uno que plantea la unidimensionalidad del instrumento, y otro que asume dos factores de primer orden compuestos por los ítems enunciados positiva y negativamente, respectivamente, y un tercer factor de segundo orden explicado por los dos factores de primer orden. En la Tabla N. ${ }^{\circ} 5$, se presentan los resultados de la aplicación del análisis factorial confirmatorio para cada uno de los modelos propuestos. Ambos modelos presentan índices de bondad de ajuste aceptables y tan solo el índice del error de aproximación cuadrático medio del modelo trifactorial $(\mathrm{RMSR}=0,09)$ presenta un valor fuera del rango recomendado de 0,08, aunque muy próximo al mismo. Los índices GFI y NFI en ambos casos se sitúan en torno a 0,90 por lo que han de ser considerados como satisfactorios. No obstante, la magnitud de los coeficientes estructurales alcanzan mayor peso en el modelo trifactorial, en torno a una media de 0,82 frente a la medida de 0,71 que se observa en el modelo unidimensional. 
Tabla N. ${ }^{0}$ 5. Análisis factorial confirmatorio de la escala de satisfacción matrimonial.

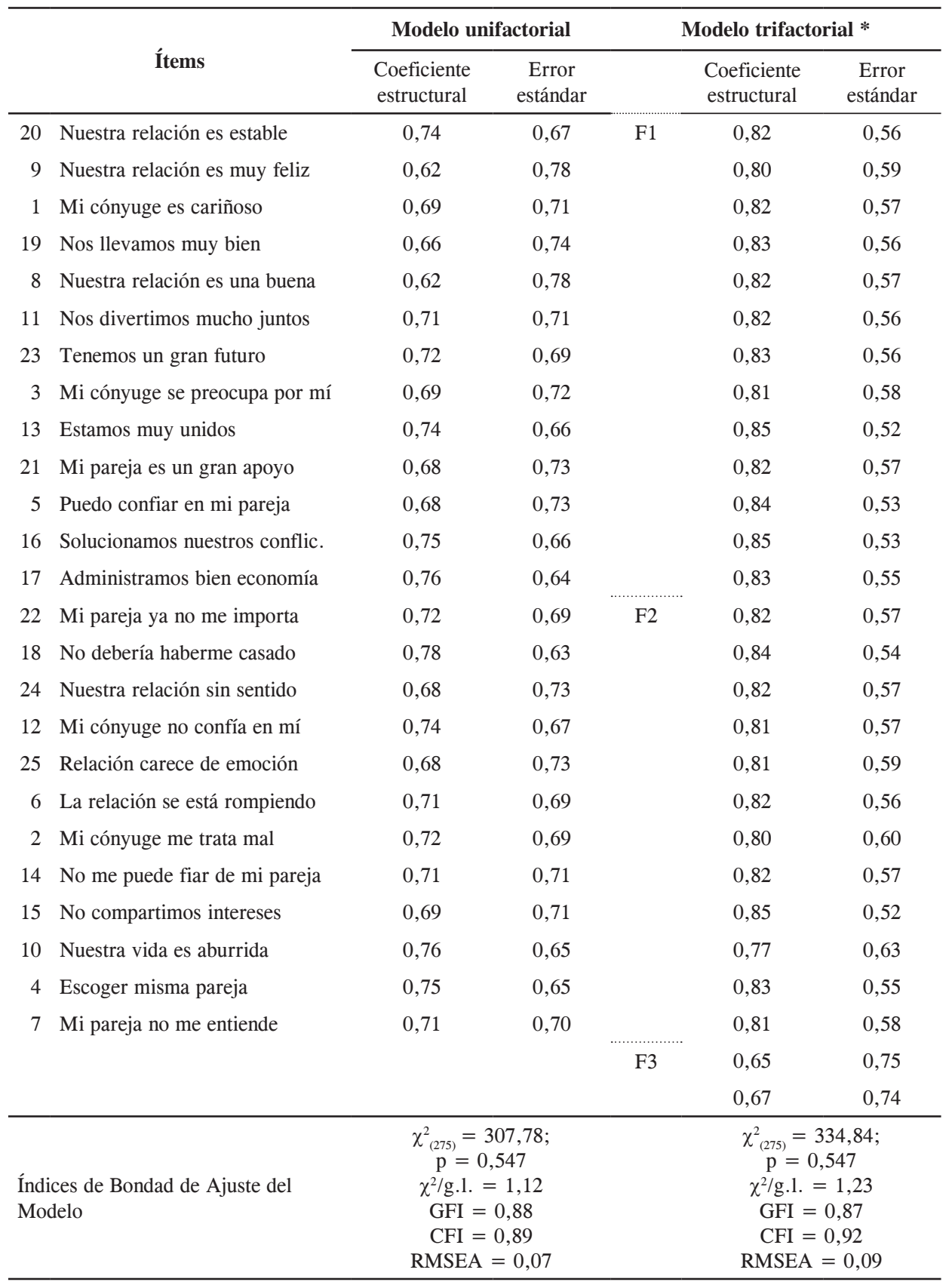

* - Modelo Trifactorial: dos factores de $1^{\circ}$ orden -F1 y F2- y uno de $2^{\circ}$ orden -F3- 
Si bien ambos modelos muestran ajustes apropiados entre la estructura empírica de los datos y los modelos teóricos, asumimos que el modelo trifactorial es más completo que el unidimensional ya que asume a aquél en su factor de segundo orden y alcanza un mejor ajuste. El pequeño desvío observado en el índice RMSR pudiera ser atribuido a cuestiones relacionadas con el tamaño de la muestra, por lo que se sugiere probar este modelo tridimensional con una muestra más extensa. Por el momento, para próximos análisis en este informe, asumiremos un indicador global de la escala tomando para su cálculo la contribución de todos sus componentes.

Con el fin de analizar la validez concurrente del ISM, se estudió su correlación con otros instrumentos afines (Tabla N. ${ }^{\circ} 3$ ). En lo que se refiere a la escala ASPA se observan unas correlaciones elevadas sobre todo con tres de las subescalas: agresión activa $(0,56)$, agresión pasiva $(0,60)$ y aserción $(-0,50)$. Es decir, a mayor puntuación en insatisfacción, mayor tendencia al empleo de la agresión como medio de expresión, ya sea directa y menor asertividad y menor aserción, es decir, menor empleo de expresión asertiva de sentimientos. También la satisfacción familiar tiene una importante correlación con la satisfacción de pareja; a mayor insatisfacción con la pareja menor satisfacción experimentada a nivel familiar. Respecto a su asociación con la SLH-30, se observan asociaciones positivas entre inestabilidad y la presencia de sintomatología (somatización, depresión, ansiedad, alteraciones cognitivas, sensibilidad interpersonal).

\section{DISCUSIÓN}

A lo largo de los análisis desarrollados se ha podido comprobar la bondad de las escalas examinadas. Los resultados de los análisis psicométricos realizados sobre la escala de inestabilidad matrimonial muestran la elevada fiabilidad y validez de la misma. Los ítems alcanzaron una fiabilidad de 0,90 , teniendo todos ellos una alta contribución al conjunto. Así mismo, el cuestionario demostró una adecuada validez de constructo, comprobado a través de diferentes estrategias de análisis. Los análisis factoriales exploratorios arrojaron un único factor, el cual explicaba un porcentaje muy alto de la varianza $(72,2 \%)$. Este resultado fue confirmado a través de análisis estructurales que mostraron la adecuación de los datos al modelo teórico propuesto. También se pudo observar una adecuada validez concurrente, a través de las correlaciones encontradas con tres criterios diferentes: las estrategias de comunicación de pareja (ASPA), la satisfacción familiar (SF) y la sintomatología experimentada (SCL-30) por las madres. Se encontraron correlaciones elevadas entre la tendencia a la inestabilidad y el mayor empleo, a la hora de afrontar situaciones conflictivas, de agresión pasiva y activa (ASPA) directa, (empleo de coacciones, insultos, amenazas) o indirecta (manipulación, retirada del afecto, limitación de la comunicación...). Asimismo, también se ha observado una mayor inestabilidad asociada a la menor satisfacción familiar y al incremento de síntomas de somatización, depresión, ansiedad y sensibilidad personal.

Los resultados obtenidos con respecto al Índice de Satisfacción Matrimonial (ISM) permiten avalar la solidez psicométrica de esta segunda escala. El índice de fiabilidad (alpha de Cronbach) fue muy elevado $(0,97)$, con una contribución muy alta de los 25 ítems de los que está formada. También las datos demostraron una adecuada validez de constructo y concurrente. Con respecto a la validez de constructo, el análisis factorial exploratorio arrojó 
una estructura de dos factores diferenciados más por cuestiones semánticas (enunciados expresados en sentido positivo o negativo) que de constructo. El análisis confirmatorio, así lo reveló. Dados los resultados previos se comprobaron dos modelos diferentes, uno unidimensional y un segundo trifactorial, con dos factores de primer orden y uno de segundo orden que agruparía a ambos. Los resultados fueron satisfactorios para ambos casos, aunque la magnitud de los coeficientes parece decantarse hacia el modelo trifactorial, lo que llevaría a tener en cuenta la contribución de todos los ítems en una única escala.

La validez concurrente, al igual que en la escala de previa, se corroboró por las altas correlaciones encontradas entre una menor satisfacción matrimonial y un mayor empleo de agresión directa o indirecta, así como un menor empleo de expresión asertiva de sentimientos a la hora de afrontar situaciones problemáticas. A su vez también se encontraron correlaciones entre inestabilidad e insatisfacción familiar, por un lado, y, por el otro, la tendencia al desarrollo de síntomas somáticos, depresivos, ansiosos, alteraciones cognitivas y sensibilidad personal.

En resumen, desde este estudio se destacan las sólidas propiedades psicométricas que presentan ambas escalas, lo cual junto con otras características como la brevedad, la simplicidad o la facilidad de corrección e interpretación, las convierte en instrumentos muy útiles para su uso en investigación y práctica clínica. Y contribuyen a rellenar el gran vacío de instrumentos de familia y pareja existentes.

No obstante, los datos sólo pueden ser tomados como un acercamiento exploratorio, pues se ha realizado exclusivamente tomando como participantes 183 mujeres pertenecientes a un contexto normalizado. Los siguientes pasos requieren la replicación de estos datos en muestras más amplias tanto de hombres como de mujeres. También permanece como aspecto pendiente, la comprobación de la validez discriminante en población clínica.

\section{REFERENCIAS BIBLIOGRÁFICAS}

1. Behling, O. y McFillen, J.M. (2000). Translating questionnaires and other research instruments. Problems and solutions. London: Sage.

2. Bentler, P. M. (1995). EQS. Structural Equations Program Manual. Encino, CA: Multivariate Software.

3. Bentler, P. M. y Bonett, D.G. (1980). Significant test and goodness of fit in the analysis of covariance structures. Psychological Bulletin, 88, 588-606.

4. Bentler, P. M. y Wu, E. J. (1995). EQS for Windows User's Guide. Encino, CA: Multivariate Software.

5. Booth, A. y Edwards, J. (1983). Measuring marital instability. Journal of Marriage and the Family, 45, 387-393.

6. Brislin, R. W. (1986). The wording and translation of research instruments. En W. J. Lonner, y W. Berry (Eds). Field methods in cross-cultural research (pp.137-164). Beverly Hills: Sage. 
7. Browne, M. W. y Cudeck, R. (1993). Alternative ways of assessing model fit. En K. A. Bollen y J.S. Long (Eds.). Testing structural equation models (pp. 136-162). Newbury Park: Sage.

8. Calvete, E. y Villa, A. (2000). Burnout y síntomas psicológicos: modelo de medida y relaciones estructurales. Ansiedad y Estrés, 6, 117-130.

9. Carrasco, M. J. (1996). Cuestionario de aserción en la pareja. Madrid: TEA.

10. Cronbach, L. J. (1951). Coefficient alpha and the internal structure of tests. Psychometrika, 6, 297-334.

11. Derogatis, L. R., Lipman, R.S., Rickels, K., Uhlenhuth, E.H. y Covi, L. (1974). The Hopkins Symptom Checklist (HSCL): a self report symptom inventory. Behavior Science, 19, 1-15.

12. Escamilla, A. (2006). Interferencia del adolescente en la pareja de rematrimonio. Tesis Doctoral. México: Facultad de Psicología, UNAM.

13. Hambleton, R. K., Merenda, P.F. y Spielberger, C.D. (2005). Adapting educational and psychological tests for cross-cultural assessment. New Jersey: Lawrence Erlbaum.

14. Hudson, W.W. (1992). The WALMYR Assessment Scales Scoring Manual. Tempe: WALMYR Publishing.

15. Johnson, D. y Booth, A. (1990). Rural economic decline and marital quality: A panel study of farm marriages. Family Relations, 39, 2, 159-165.

16. Leone, C. y Hall, I. (2003) Self-Monitoring, marital dissatisfaction, and relationship dissolution: Individual differences in orientations to marriage and divorce. Self \& Identity, 2, 189-203.

17. Locke, H. J. y Wallace, K. M. (1959). Short marital adjustment and prediction tests: Their reliability and validity. Marriage and Family Living, 21, 251-255.

18. Martínez-Pampliega, A. (Eds) (2008). Monográfico: Monoparentalidad y divorcio. Letras de Deusto, 37(115), 13-168.

19. Martínez-Pampliega, A., Sanz, M., Iraurgi, I., Cosgaya, L., Nolte, M., Alfonso, I., Benito, A y Bernal, K. (2004). Impacto de la ruptura matrimonial en el bienestar físico y psicológico de los hijos. Estudio longitudinal en familias monoparentales a cargo de la madre. Primer informe, adecuación de los instrumentos y datos del estudio piloto. Manuscrito no publicado. Bilbao: Universidad de Deusto.

20. Matthews, L. S., Wickrama, K. A. S. y Conger, R. D. (1996). Predicting marital instability from spouse and observer reports of marital interaction. Journal of Marriage and the Family, 58, 641-655.

21. Mollerstrom, W. W., Patchner, M. A. y Milner, J. S. (1992). Family Functioning and Child Abuse Potential. Journal of Clinical Psychology, 48, 445-454.

22. Muñoz, A. (2003). Relación entre las creencias irracionales y la adaptación emocional tras la separación y el divorcio. Tesis doctoral. Bilbao: Universidad de Deusto. 
23. Norusis, M. (2007). SPSS 15.0. Guide to Data Analysis. New Jersey: Prentice Hall.

24. Nunnally, J. C. y Berstein, I. J. (1995). Teoría psicométrica (3 ${ }^{\text {a }}$ edición). México: McGraw-Hill.

25. Olson, D. H., Fournier, D. G. y Druckman, J. M. (1986). PREPARE, PREPAREMC and ENRICH inventories. ( $2^{\mathrm{a}}$ edition). Minneapolis, MN: PREPARE/ENRICH, Incorporated.

26. Olson, D. H., Steward, K. L. y Wilson, L. R. (1990). Health and stress profile (HSP), revised. Minneapolis: Profile of Health Systems.

27. Olson, D. H. y Wilson, M. (1982). Family Satisfaction. En D. H. Olson, H. I. McCubbin, H. Barnes, A. Larsen, M. Muxen y M. Wilson (eds.). Family inventories: Inventories used in a national survey of families across the family life cycle (pp. 4349). St. Paul, MN: University of Minnesota.

28. Pick, S. y Andrade, P. (1988). Desarrollo y validación de la Escala de Satisfacción Marital (ESM). Psiquiatría, 4(1), 9-20.

29. Reig-Ferrer, A., Cepeda-Benito, A. y Snyder, D.K. (2004). Utility of the spanish translation of the Marital Satisfaction Inventory - Revised in Spain. Assessment, 11, 17-26.

30. Rueter, M. A., Conger, R. y Ramisetty-Mikler, S. (1999). Assessing the benefits of a parenting skills training program: A theoretical approach to predicting direct and moderating effects. Family Relations, 48, 67-78.

31. Sanz, M. (2003), El funcionamiento familiar de los drogodependientes a lo largo de un tratamiento. Tesis doctoral. Bilbao: Universidad de Deusto.

32. Schuchts, R. A. y Witkin, S. L. (1989). Assessing Marital Change during the Transition To Parenthood. Social Casework, 70, 2, 67-75.

33. Spanier, G. B. (1976). Measuring Dyadic adjustment: New scales for assessing the quality of marriage and similar dyads. Journal of Marriage and the Family, 38, 15-28. 